\title{
The postnatal management of boys in a national cohort of bladder outlet obstruction
}

Ruth Wragg ${ }^{1}$, Ewan Brownlee ${ }^{1}$, Andy Robb ${ }^{1}$, Harish Chandran ${ }^{1}$, Marian Knight ${ }^{2}$, Liam

McCarthy ${ }^{1}$

${ }^{1}$ Department of Paediatric Urology, Birmingham Children's Hospital, UK

${ }^{2}$ National Perinatal Epidemiology Unit, University of Oxford, UK

\section{Aim}

The commonest cause of congenital bladder outlet obstruction (BOO) is posterior urethral valves (PUV). Initial treatment requires decompression using a urinary catheter, but transurethral incision, vesicostomy or supra-vesical decompression (ureterostomy) have all historically been suggested as primary treatments. There is no randomized control trial evidence to guide management. PUV boys have a $50 \%$ risk of UTI, which can be reduced to $5 \%$ by circumcision, but this is not standard practice through-out the UK.

This study aims to describe the current management, the rate of circumcision and risk of UTI in a national cohort of boys with PUV in the UK.

\section{Methods}

A national cohort of patients diagnosed with bladder outlet obstruction (BOO) were recruited (via BAPS CASS) over 1 year, ethics committee approval NRES Committee South Central Oxford A (ref: 12/SC/0416). Initial data following diagnosis were collected via a questionnaire sent to all centres who reported cases. Data are presented as number (\%), analysed by Mann-Whitney U-test and Chi-square/Fisher-Exact test, $\mathrm{p}<0.05$ taken as significant.

\section{Results}

BOO presented in 121 boys 2014-2015, 113 were PUV cases. Initial treatment involved catheter placement in $87 / 121(72 \%)$, more likely to happen in antenatal vs. postnatal vs. late $(>1 y r)$ presentations, $p<0.0001$. Polyuria occurred in 23/45(51\%), 12/48(25\%), 0/28(0\%) respectively, $p<0.0001$. Initial surgical treatment was transurethral incision (TUI) in 108/121(89\%), (did not vary with presentation), vesicostomy was performed in 2 primarily (1 urethral atresia), ureterostomy was performed twice as a secondary procedure. Circumcision was performed in 52/121(43\%), most likely to be done in antenatal 
presentation vs. postnatal vs. late, $27 / 45(60 \%), 20 / 48(42 \%), 2 / 28(7 \%)$ respectively, $\mathrm{P}<0.012$. 69 UTIs occurred in 49 patients and were the commonest in the postnatal presentation. Circumcision was associated with a reduced risk of UTI $86 \%, p<0.0001$. There was a $66 \%$ reduction in UTI risk associated with TUI alone, $p<0.01$.

There was 1 death due to pulmonary hypoplasia and renal failure associated with PUV, 2 others experienced end stage renal failure (ESRF) and required dialysis.

\section{Conclusion}

Standard treatment for BOO and PUV in the current UK cohort is initial catheterization, followed by TUI. Supravesical diversion is a rescue therapy. UTIs are common, and appear significantly reduced by circumcision, however only $43 \%$ were circumcised. Initial mortality rate is $1 \%, 1.6 \%$ present in ESRF. 


\section{Introduction}

The commonest cause of congenital bladder outlet obstruction (BOO) is posterior urethral valves (PUV). PUV are also the single commonest cause of ESRF in children with approximately a quarter progressing to end-stage renal failure in childhood [1]. Routinely, initial treatment requires decompression using a urinary catheter, and then confirmation of the diagnosis often with a micturating cystourethrogram (MCUG), or by direct visualization cystoscopically. Historically transurethral incision, vesicostomy or supra-vesical decompression (end or loop ureterostomy, or Sober pelvioureterostomy en-Y) have all been promoted as primary treatments [2][3][4][5]. There is however no randomised control trial $(\mathrm{RCT})$ evidence to guide management.

Similarly, the risk of urinary tract infection (UTI) is 50\% in PUV boys. The risk of urinary tract infection (UTI) can be reduced in these boys from $50 \%$ to $5 \%$ (a reduction of $90 \%$ ), but no RCT has ever been performed to demonstrate the efficacy of this [6][7].

This study aims to describe the current management, the rate of circumcision and risk of UTI in a national cohort of boys with BOO, mostly commonly due to PUV, in the UK.

\section{Aims and methods}

A national cohort was identified of all patients referred to participating paediatric surgical units in the UK and Ireland with suspected or confirmed posterior urethral valves from October 2014 to October 2015. The British Association of Paediatric Surgeons Congenital Anomaly Surveillance System (BAPS-CASS) was used, as has been previously described [8]. Each month, routine reporting cards were sent to nominated reporting clinicians in all paediatric surgical units in the UK and Ireland, requesting details of the number of patients presenting to their unit that month with a new diagnosis of bladder outlet obstruction, i.e. suspected or confirmed posterior urethral valves. In response to a monthly card return indicating that there had been a case of PUV, clinicians were sent a form requesting further details including antenatal course, diagnosis, associated anomalies, renal function, ultrasound results, management and other outcomes for the first 3 months after initial diagnosis. Up to three reminders were sent if the first form was not returned. Duplicate 
cases were identified and removed and participating clinicians were contacted by email or telephone to seek any missing data.

Antenatal findings and maternal demographics were recorded, including any antenatal imaging or intervention. Initial investigations and management were recorded including ultrasound appearance, micturating cystourethrogram (MCUG) appearance, and details on catheterisation, renal function (plasma creatinine), post-obstructive diuresis (polyuria) if present. Data on initial and definitive surgical procedures including cystoscopic findings, and any need for secondary surgery were recorded and including complications. Data on urinary tract infections, treatment and prophylaxis were recorded.

Patients were subdivided according to presentation into an antenatal group, a postnatal group who presented within the first year of life and those who presented later.

Efficacy of circumcision and transurethral incision (TUI) of the obstructing lesion in reducing the risk of UTI were calculated by comparing boys with and without UTI in treated and untreated groups. Sub-group analysis was performed in boys under 1 year of age, with similar duration of exposure in the treated and untreated groups.

Data are presented as number (\%) and median(range), and groups were analysed using Mann-Whitney U-test, Chi-square/Fisher-Exact test, Kruskal-Wallis test, Friedman test or Spearman's rank correlation as appropriate, $p<0.05$ taken as significant. 


\section{Results}

In this national survey 113 boys with PUV were identified, as well as 8 other boys with rare causes of congenital BOO. PUV accounted for 113 (93.4\%), a complete membrane/urethral atresia for 4, Cobbs Collar for 3 , and anterior syringocele for 1 . This was a multicentre cohort of patients contributed by 25 out of 26 (96\%) paediatric urology centres in the UK and Eire.

Initial treatment involved catheter placement in $87 / 121(72 \%)$ and was more likely to happen in boys with antenatal vs. postnatal vs. late(>1yr) presentations, 44/45(98\%) vs $35 / 48(73 \%)$ vs $8 / 28(29 \%)$ respectively, $\mathrm{P}<0.0001$, table 1 . Catheterisation was predominantly urethral $81 / 87(93 \%)$, but suprapubic catheters were placed in $6 / 87(7 \%)$. Polyuria after catheter placement occurred in 23/45(51\%) of the antenatal group, $12 / 48(25 \%)$ of the postnatal group and $0 \%$ of the late group, $\mathrm{P}<0.0001$, table 1 . The duration of polyuria (days) correlated with the plateau creatinine ( $\mu$ mols $/ \mathrm{l})(\mathrm{p}<0.01, \mathrm{rS}=0.3)$, and also varied according timing of presentation: antenatal median 2 (range 0-31) days vs postnatal 0 (0-24) days vs late $(>1 \mathrm{yr}) 0(0-0)$ days $(\mathrm{P}<0.01)$, table 1 . Peak plasma creatinine $(\mu \mathrm{mols} / \mathrm{I})$, plateau creatinine and nadir creatinine one-month post operatively were recorded, figure 1 , table 1 . The creatinine was significantly higher in the antenatal group compared to the postnatal and late presentation, for peak creatinine 136(83-208), 39(25-76), 46(3460) $\mu \mathrm{mols} / \mathrm{I}$ respectively $(P=0.0001)$, figure 1 . Similar findings were present for plateau and 1-month nadir creatinine, table 1. In the antenatal group there was a drop in creatinine progressively from peak creatinine to plateau to the one-month post-op creatinine $(P=0.0001)$. There was a similar drop from peak to plateau in the postnatal group, $(P=0.0001)$. However, no further drop occurred from plateau to one-month nadir. In the late presentation group there was no change in creatinine from peak to plateau to the onemonth nadir $(p=0.1)$, figure 1 .

Initial surgical treatment was transurethral incision (TUI) in 108/121(89\%), which did not vary with timing of presentation, table 1 . Vesicostomy was performed in two boys as a primary procedure $2 / 121(2 \%$ ) (one with urethral atresia). 4 had suprapubic catheters inserted whilst they grew to a size appropriate for TUI, 1 had a drainage of an intra- 
abdominal collection, 4 had other procedures during which PUV were found incidentally and treated (e.g. Cystoscopic treatment of bladder stones, EUA trauma to urethra). Ureterostomy was performed in two boys as a secondary procedure.

Sixty-nine UTIs occurred in 49 patients during initial follow-up (17 patients had multiple UTIs) and were commonest in the postnatal group, table 1. The risk of UTI in this national cohort was 49/121 (40\%). Circumcision was performed in 52/121(43\%). This was most likely to be done in those with antenatal presentation vs. postnatal vs. late, $27 / 45(60 \%)$, $36 / 48(42 \%)$, and $2 / 28(7 \%)$ respectively, $\mathrm{P}<0.012$.

Simple comparison of risk of UTI in circumcised compared to uncircumcised boys was performed. Circumcision reduced the odds of UTI by $87 \%, \mathrm{P}<0.001$, table 2 . The duration of follow-up and potential exposure to UTI in this analysis was 300(149-2245) days vs. 86(51151) days for uncircumcised and circumcised groups respectively, $\mathrm{P}<0.0001$. Further subgroup analysis was performed in boys under 1 year of age, selecting equal length of followup for uncircumcised and circumcised groups. Follow-up exposure times were 112(102-158) days vs. 110(81-175) days for 25 circumcised and 30 uncircumcised patients in this subgroup, $\mathrm{P}=0.5$ Mann-Whitney U-test. Circumcision reduced the risk of UTI by $92 \%$ in this subgroup analysis, $\mathrm{P}<0.01$, table 2.

There were 65 patients who had TUI alone, who were never circumcised. This meant that a comparison could be made between boys with and without UTI before and after TUI, whilst still in an uncircumcised state. Sub-groups were selected under 1 year of age, with equivalent duration of exposure for pre-op 28, and post-op 37, TUI, 81(34-187) days vs. 94(68-141) days, respectively, $\mathrm{P}=0.64$. TUI alone reduced the risk of UTI by $91 \%, \mathrm{P}<0.001$, table 3.

There was one death due to pulmonary hypoplasia and renal failure associated with his PUV.

Two others experienced ESRF: one patient presented with end stage renal failure (ESRF), another progressed to this. Both required dialysis. 


\section{Discussion}

This is the first report of the postnatal management of a national cohort of boys with PUV, and other rarer causes of congenital BOO. It describes the current management of boys with PUV, and identifies the difference in behaviour between boys with an antenatal, postnatal and late presentations. This risk of UTI in this cohort was 40\%. Circumcision was protective, with similar efficacy to previous reports [6]. The protective effect of transurethral incision of PUV has however not been described before.

This national cohort confirms that there is a consensus in the UK now that standard treatment of boys with PUV is initially antenatal diagnosis if possible, with early catheterisation to decompress the obstructed system. This may be urethral (in 93\%) or suprapubic. Post obstructive diuresis is managed with fluid resuscitation as appropriate in conjunction with the nephrologists. Diagnosis is confirmed with an MCUG in the majority of cases and transurethral incision (TUI) is the treatment of choice (89\%). Vesical and supravesical diversion are rescue therapies.

Presentation of PUV can be usefully divided into 3 groups that respond differently on initial management- antenatal (on sonographic features), postnatal (with UTIs) and late (with bladder dysfunction, UTIs and incontinence).

Although polyuria (post-obstructive diuresis) is very well recognised following catheterisation in boys with PUV, the incidence and duration of this is not well described in the literature. In the patients who present with an antenatal diagnosis, a third had a polyuric phase due to post-obstructive diuresis following initial catheterisation (which was done in $98 \%$ of this group). Post-obstructive diuresis was far less prevalent in those who presented postnatally and did not happen in the late presenters (over 1 year of age). There is a statistically significant correlation between renal dysfunction as measured by plasma creatinine and duration of polyuria (days). Creatinine was significantly raised at birth in the antenatal group but with appropriate management reduced in the majority to normal onemonth post-operatively. This is encouraging, given the prognostic value of the nadir creatinine for future renal function [9]. The postnatal and late presenters were much less likely to have a significantly raised creatinine at presentation, suggesting that these are a less severe group in the spectrum of the disease. This has also been shown to correlate with degree of hydoureteronephrosis (HUN), and renal dysplasia on initial ultrasound [10]. 
In the UK, the mainstay of treatment is cystoscopic valve ablation (TUI) previously documented to be a safe management plan with reliable outcomes [11][12][13]. Our cohort confirms this, the majority being managed initially with ablation (89\%) rather than a diversionary procedure (2\%). Other procedures that may be carried out include suprapubic catheterisation, vesicostomy and ureterostomy usually in the case of a patient who is too small to cystoscope. Some require drainage first in the context of urinary ascites or a urinoma. Of those who underwent initial valve ablation, the majority had a successful outcome, only two requiring subsequent diversion. Historical series and publications from other countries describe much higher rates of supravesical diversion (8-47\%)[12][14][15]. Vesicostomy or ureterostomy remain useful secondary procedures in the rare case that requires more than ablation.

Circumcision as a preventative procedure for UTIs is becoming more common, especially in those diagnosed antenatally. It has previously been suggested to be effective in reducing the risk of UTI [6] [16]. This national cohort supports this practice, with an $92 \%$ reduction in UTI risk post circumcision. This provides further evidence for the importance of counselling for circumcision as a combined approach with TUI for UTI prophylaxis. TUI alone appears to also be associated with a reduction in risk of developing UTIs with a reduction of $91 \%$. It occurred in boys who had not been circumcised, so is an independent effect. This has not been previously reported. There is a limitation in this current cohort with the relatively short follow up time: it is likely that the more UTIs will be experienced by these boys, and the efficacy demonstrated both by circumcision and TUI is likely to be eroded to some degree.

The prospect of mortality from PUV has progressively reduced with time. The mortality rate in the 1960 s was $20 \%$, dropping by the 1990 s to $3 \%$ [17]. The risk now is approximately $1 \%$ in a modern health-care system. Morbidity can also be significant with two patients developing end stage renal failure within the one year follow up that with have attained so far. Again, with longer follow-up it is likely that mortality and morbidity will increase. No randomised controlled trial (RCT) has ever been done to compare different treatments for boys with PUV, or other causes of $\mathrm{BOO}$, at least in part because these are rare conditions, with an incidence of $1 / 3500$ boys. Prospective national studies such as BAPS CASS are however useful methods in collating data producing useful results when RCTs 
might fail because of problems with recruitment and duration of study needed. This study raises many questions about this cohort of patients: what is their renal function long-term ( $25 \%$ are likely to progress to ESRF by the end of childhood)? What is their bladder function, and continence long-term? How many will require bladder drainage by clean intermittent catheterisation $(\mathrm{CIC})$, surgical intervention to manage their lower tract dysfunction? What is their risk of UTI as they grow up? This cohort could very usefully be studied again at 5,10 and 15 years of age, and would provide prospective data to answer many of these questions.

In conclusion, PUV remains the most common cause of BOO in children. Preoperative stabilisation, awaiting resolution of polyuria and plateauing of creatinine remains the mainstay of initial treatment. The majority can be managed successfully with TUI, and diversion is rarely indicated, except as a rescue therapy. Circumcision should be considered, along with TUI, as a tool for urinary tract infection prevention and thus preservation of renal function.

\section{Funding}

This paper presents independent research arising from a Research Professorship award to Prof Marian Knight, NIHR-RP-011-032, supported by the National Institute for Health Research. The views expressed in this publication are those of the author(s) and not necessarily those of the NHS, the National Institute for Health Research or the Department of Health. 


\section{References}

[1] Woolf AS, Thiruchelvam N. Congenital obstructive uropathy: Its origin and contribution to end- stage renal disease in children. AdvRen ReplaceTher 2001;8:157.

[2] Atwell JD. Posterior urethral valves in the British Isles: a multicenter B.A.P.S. review. J Pediatr Surg 1983;18:70-4.

[3] Casale AJ. Early ureteral surgery for posterior urethral valves. Urol Clin North Am 1990;17:361-72.

[4] Parag P, Sen S, Chacko J, Zachariah N, Thomas G, Mammen KE. Bilateral high loop ureterostomy in the primary management of posterior urethral valves in a developing country. Pediatr Surg Int 2001;17:157-9.

[5] Sober I. Pelvioureterostomy-En-Y. The Journal of Urology 1972;107:473-5..

[6] Mukherjee S, Joshi A, Carroll D, Chandran H, Parashar K, McCarthy LS. What is the effect of circumcision on risk of UTI in boys with posterior urethral valves? Journal of Pediatric Surgery 2009.

[7] Morris BJ, Wiswell TE. Circumcision and lifetime risk of urinary tract infection: a systematic review and meta-analysis. J Urol 2013;189:2118-24.

[8] Bradnock TJ, Marven S, Owen A, Johnson P, Kurinczuk JJ, Spark P, et al. Gastroschisis: one year outcomes from national cohort study. BMJ 2011;343:d6749.

[9] Coleman R, King T, Nicoara C-D, Bader M, McCarthy L, Chandran H, et al. Nadir creatinine in posterior urethral valves: How high is low enough? J Pediatr Urol 2015;11:356.e1-5.

[10] Brownlee E, Wragg R, Robb A, Knight M, McCarthy L. Current epidemiology and antenatal presentation of Posterior Urethral Valves: Outcome of BAPS CASS national audit. JPS May 2018 In Press.

[11] Smith GH, Canning DA, Schulman SL, Snyder HM 3rd, Duckett JW. The long-term outcome of posterior urethral valves treated with primary valve ablation and observation. J Urol 1996;155:1730-4.

[12] Farhat W, McLorie G, Capolicchio G, Khoury A, Bägli D, Merguerian PA. Outcomes of primary valve ablation versus urinary tract diversion in patients with posterior urethral valves. Urology 2000;56:653-7.

[13] Sarhan O, El-Ghoneimi A, Hafez A, Dawaba M, Ghali A, Ibrahiem E-H. Surgical complications of posterior urethral valve ablation: 20 years experience. J Pediatr Surg 2010;45:2222-6.

[14] Warren J, Pike JG, Leonard MP. Posterior urethral valves in Eastern Ontario - a 30 year perspective. Can J Urol 2004;11:2210-5.

[15] Narasimhan KL, Kaur B, Chowdhary SK, Bhalla AK. Does mode of treatment affect the outcome of neonatal posterior urethral valves? J Urol 2004;171:2423-6.

[16] Singh-Grewal D, Macdessi J, Craig J. Circumcision for the prevention of urinary tract infection in boys: a systematic review of randomised trials and observational studies. Arch Dis Child 2005;90:853-8.

[17] Agarwal S. Urethral valves. BJU Int 1999;84:570-8. 
Legends:

Figure 1: Progression in plasma creatinine following initial bladder catheterization and initial surgery in boys with PUV (and other rarer causes of BOO), by presentation: antenatal vs. postnatal vs. late (>1year age). (Friedman statistics used for paired data analysis).

Table 1: BAPS CASS PUV study initial management and outcome results stratified by presentation: antenatal vs. postnatal vs. late.

Table 2: Efficacy of circumcision and TUI in reduction of risk of UTI in boys with BOO. Effect of circumcision; Sub-group analysis of boys under 1 year of age, with equal duration of exposure in both circumcised and uncircumcised groups.

Table 3: Efficacy of TUI alone in reduction of risk of UTI in uncircumcised boys, with subgroup analysis for boys under 1 year of age, with equal duration of exposure in pre-op and post-op TUI groups. 


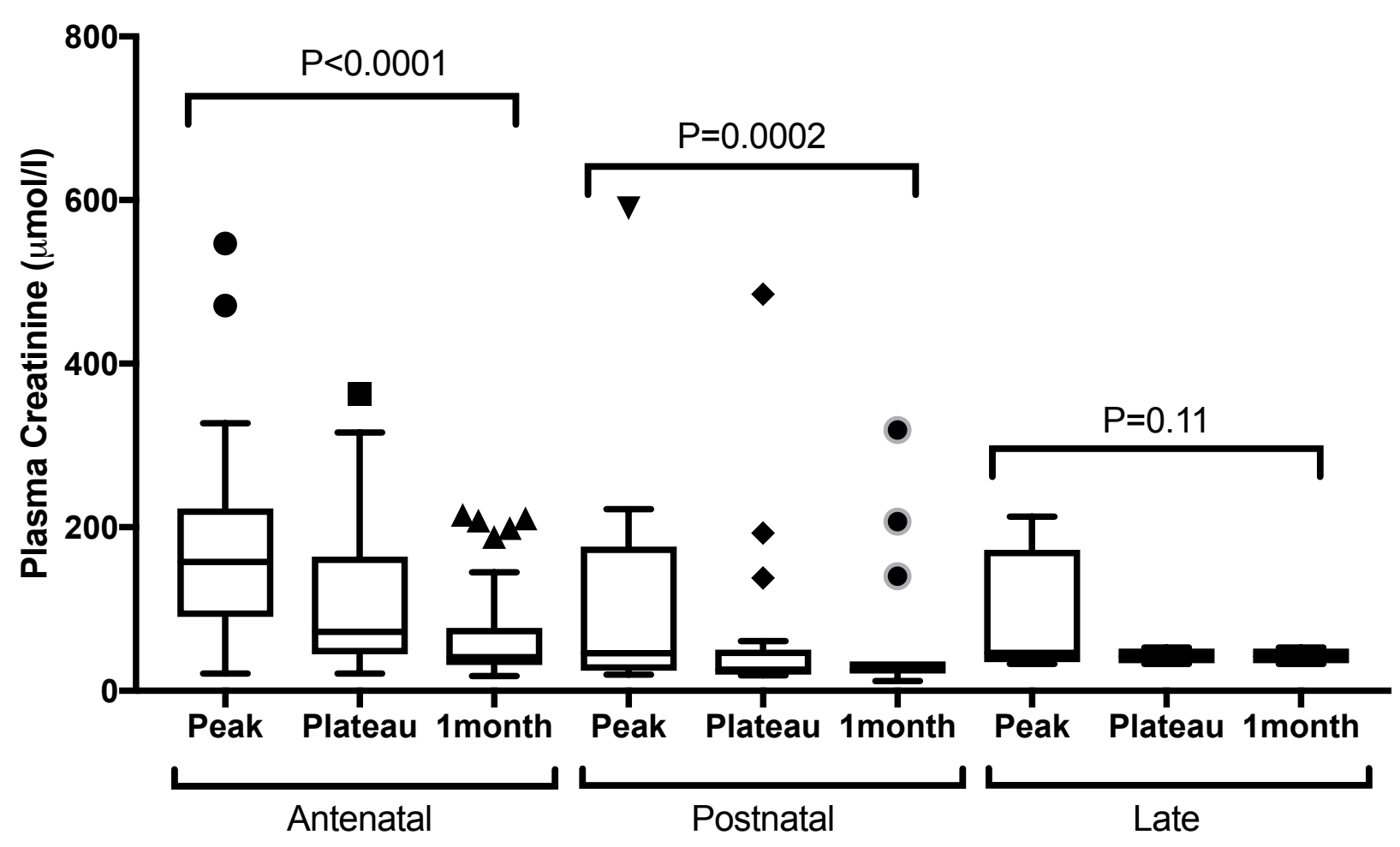


Table 1

\begin{tabular}{|c|c|c|c|c|c|}
\hline & Presentation & Antenatal & Postnatal & Late $(>1 \mathrm{yr})$ & $P$ value \\
\hline & Patients & 45 & 48 & 28 & \\
\hline \multirow{6}{*}{$\begin{array}{l}\text { Initial Management } \\
\text { and response }\end{array}$} & Catheter & $44(98 \%)$ & $35(73 \%)$ & $8(29 \%)$ & 0.0001 \\
\hline & Polyuria (\%) & $23(51 \%)$ & $12(25 \%)$ & $0(0 \%)$ & 0.0001 \\
\hline & Polyuria (days) Median (Range) & $2(0-31)$ & $0(0-24)$ & $0(0)$ & 0.01 \\
\hline & Creatinine Peak (IQR) & $136(83-208)$ & $39(25-76)$ & $46(34-60)$ & 0.0001 \\
\hline & Creatinine Plateau & $58(43-146)$ & $26(20-40)$ & $45(36-55)$ & 0.0001 \\
\hline & Creatinine 1-month nadir & $39(30-72)$ & $26(21-36)$ & $42(34-52)$ & 0.014 \\
\hline \multirow[t]{3}{*}{ Primary Surgery } & Trans-urethral incision (TUI) & $39(87 \%)$ & $46(95 \%)$ & $23(82 \%)$ & \\
\hline & Vesicostomy & $2(5 \%)$ & 0 & 0 & 0.1 \\
\hline & Ureterostomy & 0 & 0 & 0 & \\
\hline Secondary Surgery & Ureterostomy & 1 & 1 & 0 & \\
\hline \multirow{3}{*}{$\begin{array}{c}\text { Timing } \\
\text { Circumcision }\end{array}$} & With TUR & 19 & 8 & 2 & \\
\hline & Delayed & 8 & 12 & 0 & 0.012 \\
\hline & Not done & 18 & 28 & 26 & \\
\hline
\end{tabular}


Table 2

Circumcision reduces risk of UTI

\begin{tabular}{|c|c|c|c|c|c|}
\cline { 2 - 6 } \multicolumn{1}{c|}{} & & Uncircumcised & Circumcised & P-Value & Odds Ratio \\
\hline \multirow{2}{*}{ All Follow-up } & Patients & 66 & 41 & & \\
\cline { 2 - 6 } & UTIs & $25(38 \%)$ & $3(7 \%)$ & 0.0001 & 7.6 \\
\hline Sub-Group: & Patients & 25 & 32 & & \\
\cline { 2 - 6 } (Equal Exposure) & UTIs & $11(44 \%)$ & $2(6 \%)$ & 0.0011 & 11.79 \\
\hline
\end{tabular}


Table 3

TUI reduces risk of UTI in uncircumcised boys

\begin{tabular}{|c|c|c|c|c|c|}
\cline { 2 - 6 } \multicolumn{1}{c|}{} & & Pre-TUI & Post-TUI & P-Value & Odds Ratio \\
\hline \multirow{2}{*}{ Uncircumcised } & Patients & 37 & 37 & & \\
\cline { 2 - 6 } & UTIs & $14(38 \%)$ & $3(8 \%)$ & 0.0047 & 6.9 \\
\hline Sub-Group: & Patients & 27 & 25 & & \\
\cline { 2 - 6 } (Equal Exposure) & UTIs & $12(44 \%)$ & $2(8 \%)$ & 0.0011 & 10 \\
\hline
\end{tabular}

